\title{
Commentary Should dialysis be offered in all cases of metformin-associated lactic acidosis?
}

\author{
$S$ Neil Finkle
}

Capital Health, 5089 Dickson Centre, 5820 University Avenue, Halifax, Nova Scotia, Canada, B3H 1V8

Corresponding author: S Neil Finkle, neil.finkle@cdha.nshealth.ca

Published: 9 January 2009

Critical Care 2009, 13:110 (doi:10.1186/cc7161)

This article is online at http://ccforum.com/content/13/1/110

(c) 2009 BioMed Central Ltd

See related research by Peters et al., http://ccforum.com/content/12/6/R149

\begin{abstract}
Metformin is commonly used in diabetes mellitus type 2 , with lactic acidosis being a rare but potentially fatal complication of this therapy. The management of metformin-associated lactic acidosis (MALA) is controversial. Treatment may include supportive care, activated charcoal, bicarbonate infusion, hemodialysis, or continuous venovenous hemofiltration. In the previous issue of Critical Care, Peters and colleagues systematically evaluated outcomes in MALA patients admitted to their intensive care unit. The mortality rate of patients who received dialysis was similar to that of patients who were not dialyzed. However, it was the more acutely and chronically ill patients who actually received dialysis. This suggests that hemodialysis was beneficial in preventing a higher mortality rate in those who required renal replacement therapy.
\end{abstract}

The literature on the management of metformin-associated lactic acidosis (MALA) is sparse and consists of case reports and case series. In the previous issue of Critical Care, Peters and colleagues [1] presented a retrospective cohort study in patients with MALA. This study represents an important step forward in systematically evaluating outcomes in this rare but serious condition.

Metformin is commonly used in type 2 diabetes mellitus and accounts for approximately one third of all prescriptions for oral hypoglycemic agents in the US [2]. The United Kingdom Prospective Diabetes Study demonstrated impressive reductions in diabetes-related endpoints and mortality in overweight patients with type 2 diabetes who used this drug [3]. A rare but extremely serious adverse effect of this medication is lactic acidosis, which carries a staggering $50 \%$ mortality rate [4].

Metformin is renally cleared and is known to accumulate in patients with chronic kidney disease [4]. Current guidelines stipulate that it be used with caution in estimated glomerular filtration rates (eGFRs) of less than $60 \mathrm{~mL} / \mathrm{minute}$ and not at all in eGFRs of less than $30 \mathrm{~mL} /$ minute [5]. Identified risk factors for MALA include acute kidney injury (AKI), hypoxemia, sepsis, alcohol abuse, liver failure, myocardial infarction, and shock [6]. Medications that interfere with renal hemodynamic autoregulation (that is, angiotensin-converting enzyme inhibitors, angiotensin receptor blockers, and nonsteroidal anti-inflammatory drugs) and volume depletion are frequently implicated in generating the AKI leading to MALA [4]. The incidence of MALA is quoted at 1 to 5 cases per 100,000 patient-years but may be as high as 30 cases per 100,000 patient-years [4].

The mainstay of MALA therapy is supportive care. Particular attention should be paid to normalizing the acid-base imbalance, eliminating offending medication, and treating concomitant disease [4]. Activated charcoal may also have a role, especially in cases of metformin overdose [6]. Intravenous sodium bicarbonate is commonly used to correct blood $\mathrm{pH}$. Renal replacement therapies, including conventional hemodialysis and continuous venovenous hemofiltration, have been successfully employed in MALA [6-11]. These allow for both isovolemic correction of the metabolic acidosis as well as removal of metformin and lactate [4].

Peters and colleagues [1] performed a 5-year retrospective review of all patients admitted to their intensive care unit presenting with MALA. They defined MALA as lactic acidosis (lactate of greater than $5 \mathrm{mmol} / \mathrm{L}$ and bicarbonate of less than $22 \mathrm{mmol} / \mathrm{L}$ ) occurring in a patient who was chronically taking metformin or in the setting of a metformin overdose. No patients actually had MALA as their admission diagnosis. Most were admitted for management of shock or acute renal failure. MALA was part of the clinical presentation in this patient cohort rather than an admission diagnosis. MALA

$\mathrm{AKI}=$ acute kidney injury; eGFR = estimated glomerular filtration rate; MALA = metformin-associated lactic acidosis. 
accounted for $0.84 \%$ of all admissions and demonstrated a $30 \%$ mortality rate. Eighty percent of these patients developed acute renal failure and $62.5 \%$ required hemodialysis. Only one patient with normal renal function was dialyzed because of severe acidosis.

The definition of MALA in this study did not duly account for people presenting primarily with tissue hypoperfusion as the likely cause of their lactic acidosis. Although metformin may interfere with lactate clearance in a shock state, it is not thought to be the primary cause of the acidosis. Restoration of hemodynamic stability rather than dialysis is the goal of therapy in these cases. Most of the patients who died in this study were admitted with shock, suggesting that hypoperfusion, rather than metformin, was the principal cause of their lactic acidosis. However, MALA itself can present with hypotension due to negative inotropic effects and increased systemic vascular resistance with acidosis $[4,6]$.

The mortality rate in MALA was not altered by hemodialysis. This may be a reflection of the small size of this study. Upon closer inspection of the data, those patients who were dialyzed were more acutely ill as they had higher values on the SAPS II (Simplified Acute Physiology Score II). Furthermore, those who were dialyzed trended toward having a larger burden of comorbidity (Charlson index) and more severe acidosis. These data strongly suggest that hemodialysis may be of benefit in MALA. This is in keeping with the available literature.

\section{Competing interests}

The author declares that he has no competing interests.

\section{References}

1. Peters N, Jay N, Cravoisy A, Barraud D, Nace L, Bollaert PE, Gibot S: Metformin-associated lactic acidosis in intensive care unit. Crit Care 2008, 12:R149.

2. Wysowski DK, Armstrong G, Governale L: Rapid increase in the use of oral antidiabetic drugs in the United States, 1990-2001. Diabetes Care 2003, 26:1852-1855.

3. Garber MD: Metformin: mechanisms of antihyperglycemic action, other pharmacodynamic properties, and safety perspectives. Endocr Pract 1997, 3:359-370.

4. Prikis M, Mesler EL, Hood VL, Weise WJ: When a friend can become an enemy! Recognition and management of metformin-associated lactic acidosis. Kidney Int 2007, 72:11571160.

5. Canadian Diabetes Association Clinical Practice Guidelines Expert Committee: Canadian Diabetes Association 2008 clinical practice guidelines for the prevention and management of diabetes in Canada. Can J Diabetes 2008, 32:S1-S201.

6. Spiller HA, Sawyer TS: Toxicology of oral antidiabetic medications. Am J Health Syst Pharm 2006, 63:929-938.

7. Guo PY, Storsley LJ, Finkle SN: Severe lactic acidosis treated with prolonged hemodialysis: recovery after massive overdoses of metformin. Semin Dial 2006, 19:80-83.

8. DePalo VA, Mailer K, Yoburn D, Crausman RS: Lactic acidosis. Lactic acidosis associated with metformin use in treatment of type 2 diabetes mellitus. Geriatrics 2005, 60:36, 39-41.

9. Lalau JD, Westeel PF, Debussche X, Dkissi H, Tolani M, Coevoet B, Temperville B, Fournier A, Quichaud J: Bicarbonate haemodialysis: an adequate treatment for lactic acidosis in diabetics treated by metformin. Intensive Care Med 1987, 13:383-387.
10. Bruijstens LA, van Luin M, Buscher-Jungerhans PM, Bosch FH: Reality of severe metformin-induced lactic acidosis in the absence of chronic renal impairment. Neth J Med 2008, 66: 185-190.

11. Nyirenda MJ, Sandeep T, Grant I, Price G, McKnight JA: Severe acidosis in patients taking metformin-rapid reversal and survival despite high APACHE score. Diabet Med 2006, 23:432435. 